\title{
Impact of COVID-19 Pandemic on Use of Telemedicine Services in an Academic Medical Center
}

\author{
Wanting CUI ${ }^{\mathrm{a}, 1}$ and Joseph FINKELSTEIN ${ }^{\mathrm{a}}$ \\ ${ }^{a}$ Icahn School of Medicine at Mount Sinai, New York, NY, USA
}

\begin{abstract}
The COVID-19 pandemic changed the landscape of telehealth services. The goal of this paper was to identify demographic groups of patients who have used telemedicine services before and after the start of the pandemic, and to analyze how different demographic groups' telehealth usage patterns change throughout the course of the pandemic. A de-identified study dataset was generated by querying electronic health records at the Mount Sinai Health System to identify all patients. 129,625 patients were analyzed. Demographic shifts in patients seeking telemedicine service were identified. There was significant increase in the middle age and older population using telehealth services. During the pandemic use of telemedicine services was increased among male patients and racial minority patients. Furthermore, telehealth services had expanded to a broader spectrum of medical specialties.
\end{abstract}

Keywords. Telemedicine, logistic regression, COVID-19

\section{Introduction}

Telemedicine has been gaining popularity in recent years, as it is an effective way to expand medical services to remote areas and to reduce medical costs $[1,2]$. Health care systems had successfully incorporated telemedicine in their treatment and management plans, such as diabetes management, postoperative care and cancer care [3 - 5]. Since the start of the COVID-19 pandemic, some cities and regions including New York City had issued 'stay at home' orders resulting in increased number of patients receiving medical advice through telemedicine service. Mount Sinai Health System has been offering secure outpatient video visits integrated into the patient portal of an electronic health record before and during the pandemic however a comparative temporal analysis of these services has not been conducted systematically. It is imperative to analyze characteristics of increased services due to a pandemic since ability to expand these services to more medical disciplines and populations are crucial for successful health care delivery during a pandemic. The goal of this paper is to identify demographic groups of patients who received telemedicine services before and after the start of the pandemic, and to analyze how different demographic groups' telehealth usage patterns change throughout the course of the pandemic.

\footnotetext{
${ }^{1}$ Wanting Cui, Center for Biomedical and Population Health Informatics, Icahn School of Medicine at Mount Sinai, 1770 Madison Ave, $2^{\text {nd }}$ Fl, New York, NY, USA, 10035, E-mail: wanting.cui@mssm.edu
} 


\section{Method}

A de-identified study dataset was generated by querying electronic health records at the Mount Sinai Health System to identify all patients who used telemedicine services between January 2019 and July 2020. The Mount Sinai Health System has 8 hospitals with over 7,400 primary care physicians and specialists. All protected health information was removed from the dataset. The original dataset contained 147,988 patients, and over 243,000 telemedicine sessions were conducted during the year and a half period. Variables in the dataset included patients' demographics, encounter diagnosis and the corresponding care providers' primary specialty. Patients with missing values in age, sex, race and diagnosis were excluded. In addition, only patients who were 18 years or older during their first telehealth visit, and patients who identified themselves as male or female were included. 129,625 patients were included.

We defined patients who are between 18 to 39 years old as young patients, patients between 40 to 64 years old as middle age patients and patients over 65 years old as older patients. A pandemic indicator was introduced to indicate whether telemedicine services were provided before or after commencement of the pandemic. The first case of COVID19 in New York City was confirmed on 02/29/2020. Thus, services delivered before the week 61 of the study (first week of March 2020) were considered to be prior to the start of pandemic, whereas services after week 61 - after the start of pandemic. The different types of diagnoses were all coded in ICD-10 codes. We also mapped patients' primary encounter diagnosis into corresponding body systems using ICD-10 codes [6].

For patients who used the service 3 times or more, we calculated the average number of days' interval between services for each patient. For patients who regularly use telehealth service, the days between services are over 10 days. There are some patients who may use telehealth services several times, but in a short period of time. This may indicate those who required frequent follow up for a single medical issue. Thus, we defined patients who frequently and regularly use telehealth services as patients who have used the service at least 3 times and the average interval between services is at least 14 days.

We performed multiple logistic regressions on 3 subsets: all patients, patients prior to the start of pandemic and patients after the start of pandemic. Age, sex and race were independent variables. In all patients, patients who had completed information were included. We used the pandemic indicator (prior: 0, after: 1) as the dependent variable. In patients prior to the start of pandemic, only patients who had used telemedicine prior the start of pandemic were included and we used the frequency indicator (infrequent: 0 , frequent: 1) as the dependent variable. In patients after the start of pandemic, only patients who enrolled in telemedicine service after the start of the pandemic were included and we used the frequency indicator (infrequent: 0, frequent: 1) as the dependent variable.

\section{Results}

2,053 patients used the service prior to the pandemic, and 127,572 patients started using the telemedicine service after the pandemic started. Of the patients who have used the service prior to the pandemic, over $95 \%(n=1,962)$ of them continued using the service during the pandemic. Over $30 \%(n=647)$ of patients who have used the service prior than the pandemic, were frequent telehealth users. However, this number decreased to 
$8 \%(n=10,398)$ for patients who started to use the telehealth service after the start of the pandemic.

The average age of telemedicine users was $40.76 \pm 14.09$ years old prior to the pandemic. This number increased to $49.67 \pm 17.72$ years old after the start of pandemic. According to Figure 1, before the start of the pandemic, the majority of patients who used the telehealth services were young patients (53.68\%). In contrast, less than $7 \%$ of patients prior to the pandemic were older adults. After the start of pandemic, the average number of telehealth sessions per day increased for all age groups. Around $35 \%$ of patients were young patients, $42 \%$ were middle age patients and $23 \%$ were older patients. In addition, over $60 \%(n=78786)$ of telemedicine users were female. Furthermore, there were significantly less Black telemedicine users (10.92\%) compared to Black in person users (around 33\%).

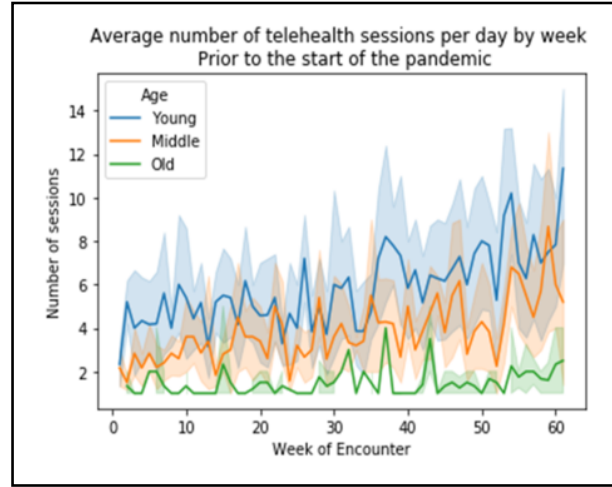

Figure 1A. Prior to the start of pandemic

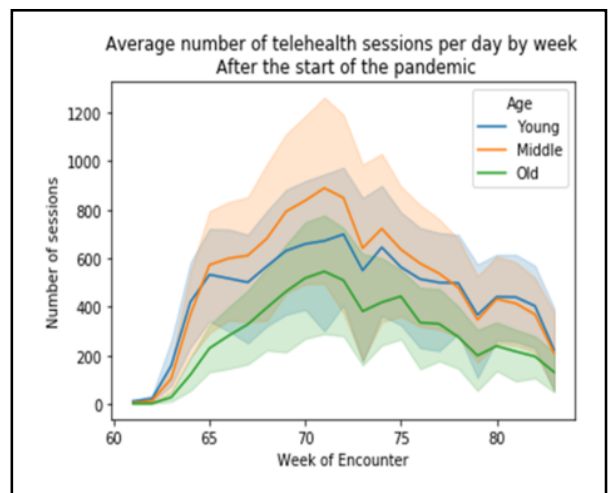

Figure 1B. After the start of the pandemic

According to logistic regression (Table 1), after the start of the pandemic, male patients, middle-age or older patients were significantly more likely to use the telemedicine service. In addition, there was an increase of non-white patients in telemedicine. Prior to the start of pandemic, age and sex were not significant variables in determine frequent telemedicine users. In contrast, after the start of pandemic, older patients, female patients and Black patients had higher odds of becoming frequent telemedicine users.

Table 1. Logistic regression results

\begin{tabular}{|c|c|c|c|c|c|c|}
\hline $\begin{array}{l}\text { Dependent } \\
\text { Variable }\end{array}$ & \multicolumn{2}{|c|}{ Pandemic Indicator } & \multicolumn{4}{|c|}{ Frequency Indicator } \\
\hline \multirow[t]{2}{*}{ Subset } & \multicolumn{2}{|c|}{ All Patients } & \multicolumn{2}{|c|}{ Prior to Pandemic } & \multicolumn{2}{|c|}{$\begin{array}{l}\text { After the start of } \\
\text { Pandemic }\end{array}$} \\
\hline & OR & P-Value & OR & P-Value & OR & P-Value \\
\hline Age Young & 1 & & 1 & & 1 & \\
\hline \multirow{2}{*}{ Age Middle } & 1.58 & 0 & 0.87 & \multirow{2}{*}{0.169} & 1.03 & \multirow{2}{*}{0.16} \\
\hline & $(1.44,1.73)$ & 0 & $(0.71,1.06)$ & & $(0.99,1.08)$ & \\
\hline \multirow{2}{*}{ Age Old } & 5.25 & 0 & 0.86 & \multirow{2}{*}{0.434} & 1.14 & \multirow{2}{*}{0} \\
\hline & $(4.40,6.26)$ & 0 & $(0.59,1.26)$ & & $(1.08,1.20)$ & \\
\hline Sex Female & 1 & & 1 & & 1 & \\
\hline Sex Male & 1.14 & 0.004 & 0.88 & 0.191 & 0.89 & 0 \\
\hline
\end{tabular}




\begin{tabular}{|c|c|c|c|c|c|c|}
\hline & $(1.04,1.25)$ & & $(0.72,1.07)$ & & $(0.86,0.93)$ & \\
\hline Race White & 1 & & 1 & & 1 & \\
\hline \multirow{2}{*}{ Race Asian } & 1.23 & \multirow{2}{*}{0.028} & 0.73 & \multirow{2}{*}{0.121} & 0.94 & \multirow{2}{*}{0.178} \\
\hline & $(1.02,1.47)$ & & $(0.49,1.09)$ & & $(0.86,1.03)$ & \\
\hline \multirow{2}{*}{ Race Others } & 1.54 & \multirow{2}{*}{0} & 0.77 & \multirow{2}{*}{0.015} & 0.81 & \multirow{2}{*}{0} \\
\hline & $(1.39,1.70)$ & & $(0.62,0.95)$ & & $(0.77,0.85)$ & \\
\hline Race Black & $\begin{array}{r}1.97 \\
(1.65,2.34)\end{array}$ & 0 & $\begin{array}{r}0.91 \\
(0.63,1.32)\end{array}$ & 0.635 & $\begin{array}{r}1.25 \\
(1.17,1.32)\end{array}$ & 0 \\
\hline
\end{tabular}

In Table 2, prior to the pandemic, patients used telemedicine for mental disorders and diseases of digestive system. After the start of pandemic, medical issues shifted to metabolic diseases and immunity disorders, diseases of the circulatory system, diseases of the skin and subcutaneous tissue, and diseases of the musculoskeletal systems. Furthermore, patients with mental disorder, diseases of digestive system and musculoskeletal systems would routinely and frequently use telemedicine services.

Table 2. Summary of body systems.

\begin{tabular}{lrrrr}
\hline & \multicolumn{2}{c}{ Pandemic Indicator } & \multicolumn{2}{c}{ Frequency Indicator } \\
\hline Body System & \multicolumn{1}{c}{ Prior } & After & Frequent & Infrequent \\
\hline 1. Infectious and parasitic disease & $3.79 \%$ & $2.93 \%$ & $2.47 \%$ & $3.06 \%$ \\
\hline 2.Neoplasms & $0.63 \%$ & $4.91 \%$ & $5.84 \%$ & $4.58 \%$ \\
\hline $\begin{array}{l}\text { 3. Endocrine, nutritional, and metabolic diseases and } \\
\text { immunity disorders }\end{array}$ & $4.7 \%$ & $9.07 \%$ & $7.86 \%$ & $9.28 \%$ \\
\hline 4. Diseases of blood and blood-forming organs & $0.08 \%$ & $0.9 \%$ & $1.04 \%$ & $0.84 \%$ \\
\hline 5. Mental disorders & $24.03 \%$ & $13.6 \%$ & $16.03 \%$ & $13.21 \%$ \\
\hline 6. Diseases of the nervous system and sense organs & $7.54 \%$ & $8.2 \%$ & $8.58 \%$ & $8.09 \%$ \\
\hline 7. Diseases of the circulatory system & $1.69 \%$ & $7.29 \%$ & $5.97 \%$ & $7.51 \%$ \\
\hline 8. Diseases of the respiratory system & $6.4 \%$ & $5.83 \%$ & $5.4 \%$ & $5.95 \%$ \\
\hline 9. Diseases of the digestive system & $30.58 \%$ & $5.69 \%$ & $8.15 \%$ & $5.62 \%$ \\
\hline 10. Diseases of the genitourinary system & $2.1 \%$ & $4.59 \%$ & $3.78 \%$ & $4.74 \%$ \\
\hline 11. Complications of pregnancy, childbirth, and the & & & & \\
puerperium & $0.18 \%$ & $0.86 \%$ & $0.95 \%$ & $0.82 \%$ \\
\hline 12. Diseases of the skin and subcutaneous tissue & $1.85 \%$ & $5.18 \%$ & $3.86 \%$ & $5.44 \%$ \\
\hline 13. Diseases of the musculoskeletal system & $2.33 \%$ & $7.39 \%$ & $9.24 \%$ & $6.81 \%$ \\
\hline 14. Congenital anomalies & $0.23 \%$ & $0.56 \%$ & $0.46 \%$ & $0.58 \%$ \\
\hline $\begin{array}{l}\text { 15. Certain conditions originating in the perinatal } \\
\text { period }\end{array}$ & $0.05 \%$ & $0.09 \%$ & $0.07 \%$ & $0.09 \%$ \\
\hline 16. Symptoms, signs, and ill-defined conditions & $8.88 \%$ & $14.09 \%$ & $13.56 \%$ & $14.1 \%$ \\
\hline 17. Injury and poisoning & $1.32 \%$ & $2.18 \%$ & $1.74 \%$ & $2.27 \%$ \\
\hline $\begin{array}{l}\text { 18. Factors influencing health status and contact with } \\
\text { health services }\end{array}$ & $3.39 \%$ & $6.49 \%$ & $4.9 \%$ & $6.83 \%$ \\
\hline Body System None & $0.25 \%$ & $0.18 \%$ & $0.11 \%$ & $0.2 \%$ \\
\hline
\end{tabular}




\section{Discussion}

The number of telehealth sessions increased drastically at the start of the pandemic. There was significant increase in middle age and older population using telehealth service. In addition, there was also increase in male patients and non-white patients. Furthermore, telehealth services had expanded to more medical specialties.

In terms of telehealth usage frequency, traditionally half of the telehealth users were frequent or routine users. After the start of the pandemic, we observed increases in both frequent and non-frequent telehealth users, with the increase in non-frequent users being much greater. Older patients and black patients were more likely to become frequent telehealth users after the pandemic. In addition, patients with mental health problems, digestive track diseases and diseases of the musculoskeletal system were likely to use the telehealth services routinely.

However, these results were periodic. As the pandemic is ongoing, we expect more changes in telehealth services will occur based on the severity of the pandemic and constant updates of telemedicine technologies. We will continue to monitor the trends of telemedicine usage and patients' retention rate.

\section{Conclusion}

The COVID-19 pandemic changed the landscape of telehealth services. Demographic shifts in patients seeking telemedicine service as well as medical specialties expansion were identified in telehealth service use after the start of the pandemic. Age, race and gender were all important factors to consider creating better telehealth services in the future. Further expansion of telemedicine services to support physical and cognitive rehabilitation [7-8], behavioral health [9], and eldercare [10-11] is warranted.

\section{References}

[1] Waller M, Stotler C. Telemedicine: a Primer. Curr Allergy Asthma Rep. 2018 Aug; 18(10):54.

[2] Finkelstein J, Friedman RH. Potential role of telecommunication technologies in the management of chronic health conditions. Dis Manag Heal Outcomes 2000;8(2):57-63.

[3] Sirintrapun SJ, et al. Telemedicine in Cancer Care. Am Soc Clin Oncol Educ Book. 2018 May;38:540545.

[4] Williams, A. M., Bhatti, U. F., Alam, H. B., \& Nikolian, V. C. The role of telemedicine in postoperative care. mHealth 2018 May; $4: 11$.

[5] Storch K, Graaf E, Wunderlich M, Rietz C, Polidori C, Woopen C. Telemedicine-Assisted SelfManagement Program for Type 2 Diabetes Patients. Diabetes Technol Ther. 2019 Sep;21(9):514-521.

[6] Ho CH, Chen YC, Chu CC, Wang JJ, Liao KM. Age-adjusted Charlson comorbidity score is associated with the risk of empyema in patients with COPD. Medicine (Baltimore). 2017 Sep;96(36),e8040.

[7] Finkelstein J, Jeong IC, Doerstling M, et al. Usability of Remote Assessment of Exercise Capacity for Pulmonary Telerehabilitation Program. Stud Health Technol Inform. 2020 Nov;275:72-76.

[8] Jeong IC, Liu J, Finkelstein J. Factors Affecting Adherence with Telerehabilitation in Patients with Multiple Sclerosis. Stud Health Technol Inform. 2019;257:189-193.

[9] Pradhan AM, Park L, Shaya FT, Finkelstein J. Consumer Health Information Technology in the Prevention of Substance Abuse: Scoping Review. J Med Internet Res. 2019 Jan;21(1):e11297.

[10] Bedra M, Finkelstein J. Feasibility of post-acute hip fracture telerehabilitation in older adults. Stud Health Technol Inform. 2015;210:469-73.

[11] Jeong IC, Finkelstein J. Interactive resistance chair to promote strengthening exercise in older adults. Stud Health Technol Inform. 2015;210:205-9. 